\title{
Analysis of Peripherally Inserted Central Catheter Line in Cancer Patients: A Single-Center Experience
}

\author{
Sulav Sapkota ${ }^{1} \quad$ Raju Sannur $^{1} \quad$ Radheshyam Naik $^{1}$ \\ ${ }^{1}$ Department of Medical Oncology and Hematology, Healthcare \\ Global Enterprises Limited, Bengaluru, Karnataka, India
}

Address for correspondence Sulav Sapkota, MD, Fellowship in Hematology and Bone Marrow Transplantation, Department of Medical Oncology and Hematology, Healthcare Global Enterprises Limited, Bengaluru, 560027, Karnataka, India (e-mail: dr.sulavsapkota@yahoo.com).

South Asian J Cancer 2021;9:253-256.

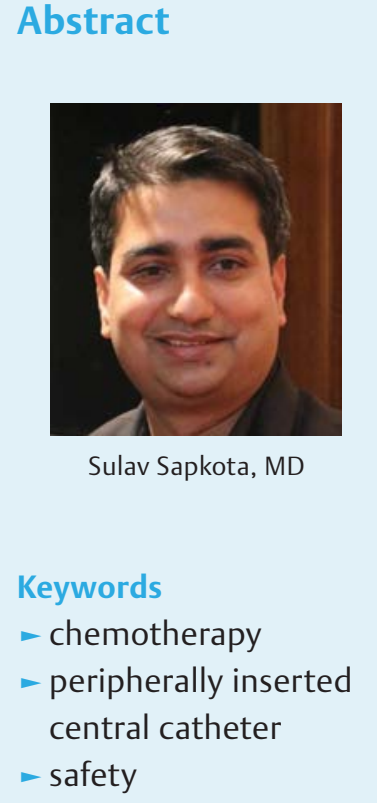

Background Peripherally inserted central venous catheters are now widely used in cancer patients who require long-term treatment, for delivering multiple infusates. We aimed to evaluate the overall use of peripherally inserted central catheter (PICC) line in cancer patients, with the objective to study the demographic profile, complications, and safety related to PICC line in cancer patients.

Methods All the patients undergoing treatment for hematological and solid malignancies with PICC line inserted at the Healthcare Global Hospital during the study were evaluated prospectively. The complications related to PICC and its safety were analyzed.

Results Five hundred PICCs were inserted over a period of 2 years to 8 months for a total of 62,440 catheter days (mean of 24 days, i.e., 4.2 months, range: 1-434 days). The most common indication for PICC was for delivering chemotherapy (100\%). Of these, 51 (10.2\%) PICCs had complications at the rate of 0.82/1000 PICC days, and hence, 41 PICCs were removed. Hematological malignancies had more complications as compared with those with solid malignancies.

Conclusions PICCs are comparatively safe method for the central venous access in cancer patients.

\section{Introduction}

Peripherally inserted central catheter (PICC) is one of the vascular access devices (VADs) that can be used for a prolonged period of time and is useful for chemotherapy infusions, antibiotic therapy, and blood transfusion as well as total parenteral nutrition. It is inserted in a peripheral vein in the cubital fossa, either the cephalic, basilic, or brachial vein and then advanced toward the heart, till the tip reaches the distal superior vena cava or cavoatrial junction. It was first described in $1975,{ }^{1}$ as an alternative to central venous catheters such as subclavian catheters, internal jugular, or femoral catheter, which have shown to have higher rate of infections.

Subclavian and jugular vein catheters might result in pneumothorax, while due to the method of placement,
PICCs have shown to have comparatively lesser complications. The PICC catheter is either made up of silicon rubber or polyurethane, where silicon rubber has a lower risk of thrombosis. ${ }^{2}$

Indications, contraindications, and potential complications must be considered prior to the insertion of a PICC. With advancing age and increasing comorbidities in our modern society, adequate venous access can sometimes be very difficult to establish. PICCs provide reliable and safe intravenous access in a variety of indications. ${ }^{3}$ One advantage of PICCs is that, it can be used for any infusate, irrespective of $\mathrm{pH}$, osmolality, or other chemical properties of the solution or medication. Many intravenous medications and solutions can cause damage to the peripheral venous endothelium and should be administered centrally to avoid this damage. ${ }^{4}$ Many
DOI https://doi.org/10.1055/s-0040-1721175 ISSN 2278-330X.

How to cite this article: Sapkota S, Sannur R, Naik R. Analysis of Peripherally Inserted Central Catheter Line in Cancer Patients: A SingleCenter Experience. South Asian J Cancer 2021;9(4):253-256. (c) 2020. Medlntel Services Pvt Ltd.

This is an open access article published by Thieme under the terms of the Creative Commons Attribution-NonDerivative-NonCommercial-License, permitting copying and reproduction so long as the original work is given appropriate credit. Contents may not be used for commercial purposes, or adapted, remixed, transformed or built upon. (https://creativecommons.org/licenses/by-nc-nd/4.0/)

Thieme Medical and Scientific Publishers Pvt. Ltd., A-12, 2nd Floor,

Sector 2, Noida-201301 UP, India 
intravenous medications and solutions can cause damage to the peripheral venous endothelium and should be administered centrally to avoid this damage. ${ }^{4}$ There is high blood flow $(\sim 21 / \mathrm{min})$ around the central terminal tip of the PICC catheter that causes immediate dilution of the infusate, which helps to protect the vessel wall from chemical irritation.

Although PICCs can remain in situ for extended periods of time, ranging from 7 days to 1 year, there is no established dwell time for PICCs, ${ }^{1,5}$ but if the therapy is expected to last longer than 1 year, a more permanent type of central access device should be considered, such as a tunneled catheter or implanted port. However, PICCs offer certain advantages over other forms of long-term VAD. ${ }^{6}$

We conducted this study to analyze the overall use of PICCs in cancer patients attending our hospital.

\section{Study Design and Methods}

This prospective study was conducted over a period of 3 years and 6 months from June 2015 to December 2018 at our tertiary comprehensive cancer hospital. A 4-Fr single-lumen triple-valved PICC was inserted under strict aseptic conditions and ultrasonography guidance. The PICCs were inserted in any of the major veins of the upper extremity and secured by stat-lock adhesive dressing and sutures. We prospectively studied and followed all patients in the insertion period, who had a PICC successfully inserted during the study, from June 15, 2015, to February 15, 2018, that is, 2 years and 8 months and maintained a pro forma. The last 10 months of the study period was dedicated for the follow-up of these patients. Those patients with histologically-proven hematological or solid malignancies were included in the study, while those with bleeding and clotting disorders were excluded. Weekly follow-up for these patients was arranged with PICC team in the ward or minor operation theater for the care of the VAD.

Written informed consent was obtained from all the patients enrolled in the study. This study was approved by the Institutional Review Board.

\section{Statistical Analysis}

The pro forma included the details of the patient and all the parameters related to PICC line such as name, age, sex, diagnosis, patient status, date of insertion and removal of PICC, time taken for insertion, length and depth of PICC line, number of attempts for insertion, type of vein accessed, complication (if any), and PICC dwell days. The data were analyzed using SPSS software version 20. Demographic characteristics were summarized by descriptive statistics. The rates of complications were expressed by percentage and per 1,000 catheter days. For the analysis, each PICC placement was counted as a new event. In the case, where patients lost to follow-up, the final visit was taken as the date of removal of PICC line.

\section{Observation and Results}

All the PICC lines were inserted by a trained person under ultrasound guidance and strict aseptic condition in minor operation theater. The mean number of attempts for skin puncture was 1.2 (range: 1-4), and the mean duration of time for PICC insertion from skin prick to fixation of PICC was 26.5 minutes (range: 6-120 minutes).

A total of 500 PICCs were inserted successfully during the study. PICC was inserted thrice to two of the patients and twice to ten patients. Five PICCs (1\%) were lost to follow-up. The demographic profile of patients and the details of PICC placement are listed in - Table 1. Overall, the patient population included 245 (49\%) men and 255 (51\%) women, with a mean age of 48 years (range: 7-83 years). Three hundred and three patients (60.6\%) had a solid malignancy and 197 (39.4\%) had hematological malignancy. Among the solid tumors, the most common were gastrointestinal tract malignancies (33\%), while lymphoma was the most common (42\%) in the hematological malignancies. The basilic vein was used most frequently, 422 times (84.4\%) for PICC placement. All patients (100\%) were discharged from hospital with a PICC in situ.

All the 500 PICCs were in place for a total of 62,440 catheter days (mean of 124 days, i.e., 4.2 months; range: 1-434 days). The most common indication for PICC was chemotherapy (100\%). The most frequently used chemotherapy regimen was adjuvant chemotherapy for gastrointestinal cancer in solid cancers and induction chemotherapy for lymphoma in hematological cancers. Majority of patients preferred PICC in this section.

A total of 51 (10.2\%) complications, at the rate of $0.8 / 1000$ catheter days, were observed during our study, out of which 17 (3.4\%) infective complications (-Fig. 1) including both superficial infection (phlebitis) and catheter-related bloodstream infections (CRBSIs) were the most common complications, while $3 \%$ was due to thrombotic event. We observed that out of the 500 PICCS removed, 22 PICCs (4\%) were removed due to death of the patients caused by progression of the disease. PICCs in 41 (8\%) patients were removed due to complications and 431 (86.2\%) PICCs were removed at the completion of therapy without any evidence of complications. We noticed that those patients with PICCs with hematological malignancies (11.6\%) were found to have more complications than solid malignancies (9.2\%). We did not observe any death due to the complications caused by PICC line.

\section{Discussion}

PICC is now emerging as one of the safe and efficacious methods of VADs. Although it has been used increasingly in majority of the cancer centers for the administration of chemotherapy drugs in India, still, the review of literature in the present work shows limited number of published articles. Hence, our attempt in this prospective study is to share our institutional experience with PICC line.

In the current study, patients were in between 7 and 84 years of age, with a mean of 48 years. Our comprehensive tertiary cancer center is well equipped with the facilities to deal with almost every cancer; hence, we observed a wider range of the age of the patients in our study. This was in contrast to the findings of Haider et al, ${ }^{7}$ where the patient age group was between 14 and 40 years. In the present study, 
Table 1 Characteristics of the patients receiving a peripherally inserted central catheter $(n=500)$

\begin{tabular}{|c|c|}
\hline Parameters & Number of patients (\%) \\
\hline \multicolumn{2}{|l|}{ Demographic profile } \\
\hline Age (mean in year) & 48 \\
\hline \multicolumn{2}{|l|}{ Sex } \\
\hline Male & $245(49)$ \\
\hline Female & $255(51)$ \\
\hline \multicolumn{2}{|l|}{ Categorization of cancer } \\
\hline Overall solid tumors & $303(60.6)$ \\
\hline Breast cancer & $85(17)$ \\
\hline GIT and PB tract & $103(20.6)$ \\
\hline Head and neck & $41(8.2)$ \\
\hline Gynecological & $31(6.2)$ \\
\hline Lung cancer & $16(3.2)$ \\
\hline Others & $27(5.4)$ \\
\hline Overall hematological cancer & $197(39.4)$ \\
\hline AML & $50(10)$ \\
\hline Lymphoma & $84(16.8)$ \\
\hline ALL & $30(6.4)$ \\
\hline Multiple myeloma & $17(3.4)$ \\
\hline Others & $16(3.2)$ \\
\hline \multicolumn{2}{|l|}{ Site of PICC line insertion } \\
\hline Right basilic vein & $87(16.7)$ \\
\hline Left basilic vein & $335(67)$ \\
\hline Right cephalic vein & $5(0.2)$ \\
\hline Left cephalic vein & $31(6.2)$ \\
\hline Right brachial vein & $19(3.8)$ \\
\hline Left brachial vein & $23(4.6)$ \\
\hline Overall complications & $51(10.2)$ \\
\hline CRBSI & $8(1.6)$ \\
\hline Phlebitis & $9(1.8)$ \\
\hline Blockage & $6(1.2)$ \\
\hline Accidental removal & $3(0.6)$ \\
\hline Leakage & $5(1.0)$ \\
\hline Thrombosis & $15(3.0)$ \\
\hline Abnormal position & $4(0.8)$ \\
\hline Hematoma & $1(0.2)$ \\
\hline \multicolumn{2}{|l|}{ Total PICCs removed } \\
\hline Removal due to death & $22(4.4)$ \\
\hline Removal due to complications & $42(8.4)$ \\
\hline $\begin{array}{l}\text { Removal after completion of } \\
\text { treatment without } \\
\text { complications }\end{array}$ & $431(86.2)$ \\
\hline Lost to follow-up & $5(1.0)$ \\
\hline
\end{tabular}

Abbreviations: ALL, acute lymphocytic leukemia; AML, acute myeloid leukemia; CRBSI, catheter-related bloodstream infections; GIT, gastrointestinal tract; PB, pancreato-biliary; PICCs, peripherally inserted central catheters.

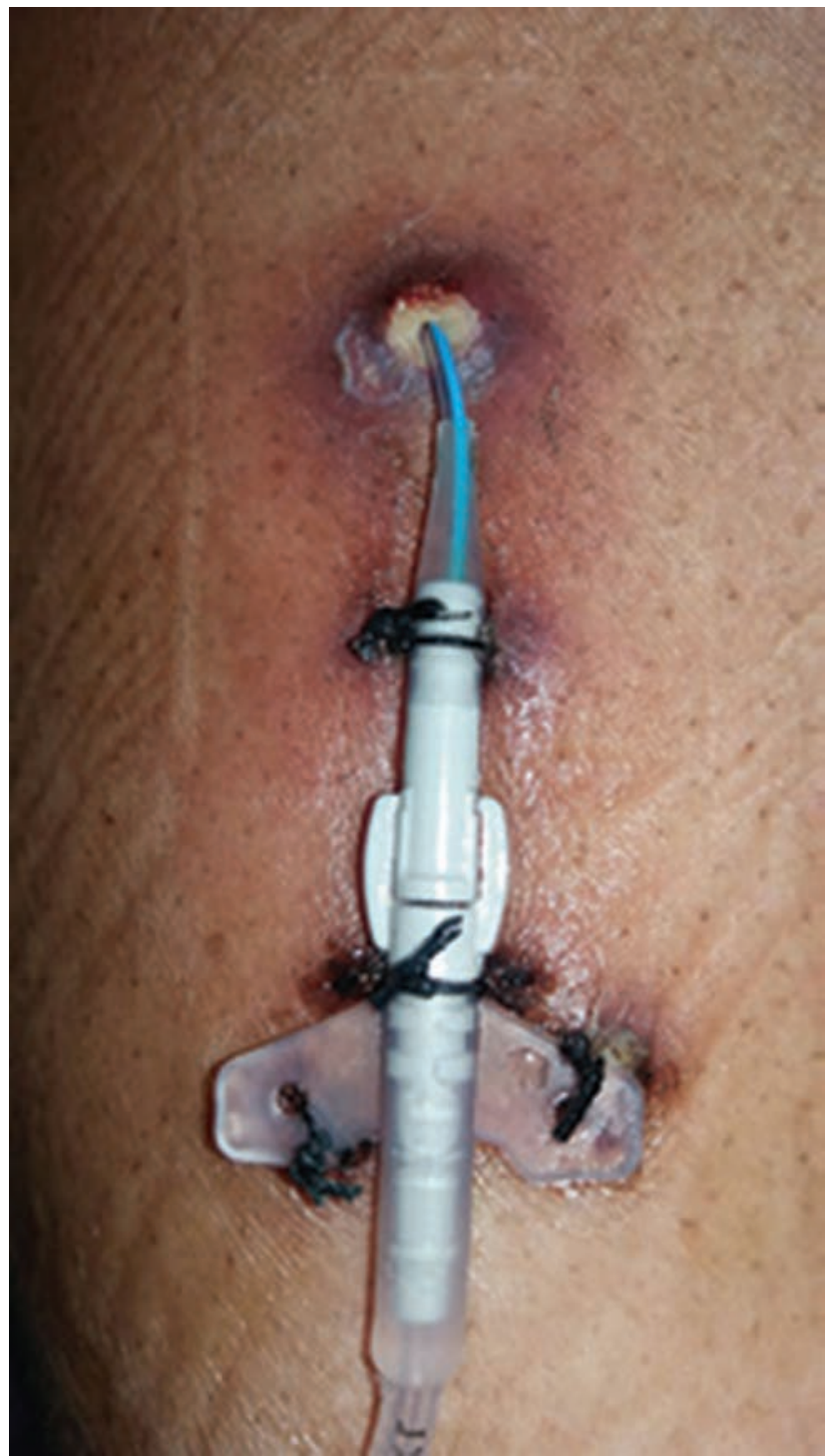

Fig. 1 Infective complication at the skin puncture site.

PICC was mainly used for delivering chemotherapy to cancer patients. We observed overall $10.2 \%$ complications in our patients, where mainly $3.4 \%$ was due to infective causes (CRBSI and phlebitis combined), while $3 \%$ was due to thrombosis. The possible explanation of infection-related complications in our setting is because of the multiple use of PICC line, use of steroids, and immunocompromised state of the patients renders them for infection. The hospital-acquired infections are another threat for developing the infective complications in these patients.

Of the 500 PICCs inserted in the present study, we observed that 431 (86.2\%) completed the respective therapies without any complications; while out of the $10.2 \%$ patients who developed complications, $8 \%$ PICCs were removed. The remaining $2.2 \%$ patients were salvaged with regular dressing and oral antibiotic, especially in cases of phlebitis. We did not observe any death due to PICC line complications, although $4 \%$ of the patients who died were because of the progression of the disease. 
The major challenge that we faced in our work was the VAD care to prevent complications. To deal with the issue, we arranged a regular follow-up for VAD care with our PICC-trained nurses, at each scheduled chemotherapy dates of the patients. We had also educated the patient attendants as well as local healthcare providers for basic VAD care and dressing. These measures increased the patient compliance with PICC line.

The considerable number of patients enrolled in the present study forms its strength as not many publications from developing nations such as India are available for the use of PICC line in cancer patients. As we did not observe any deaths related to PICC or its complications, we suggest it to be safer methods for central venous access. The shortcoming of our work is inability to study the quality of life, social impact, and financial issues related to PICC line in cancer patients.

\section{Conclusions}

Our study reinforces the notion that PICC line is safe, convenient, and is a safer mode of the central venous access in cancer patients even in Indian population. It can be used without much complication for a longer duration of time in patients needing chemotherapy. However, the quality of life, financial issues of the patients with PICC line still need to be addressed, for which more prospective studies are recommended.

\section{Funding \\ None.}

\section{Conflicts of Interest}

There are no conflicts of interest.

\section{References}

1 Hoshal VL Jr. Total intravenous nutrition with peripherally inserted silicone elastomer central venous catheters. Arch Surg 1975;110(5):644-646

2 Bishop L, Dougherty L, Bodenham A, et al. Guidelines on the insertion and management of central venous access devices in adults. Int J Lab Hematol 2007;29(4):261-278

3 Chu FS, Cheng VC, Law MW, Tso WK. Efficacy and complications in peripherally inserted central catheter insertion: a study using 4-Fr non-valved catheters and a single infusate. Australas Radiol 2007;51(5):453-457

4 Yap YS, Karapetis C, Lerose S, Iyer S, Koczwara B. Reducing the risk of peripherally inserted central catheter line complications in the oncology setting. Eur J Cancer Care (Engl) 2006;15(4):342-347

5 Walshe LJ, Malak SF, Eagan J, Sepkowitz KA. Complication rates among cancer patients with peripherally inserted central catheters. J Clin Oncol 2002;20(15):3276-3281

6 Chaitowitz I, Heng R, Bell K. Managing peripherally inserted central catheter-related venous thrombosis: how I do it. Australas Radiol 2006;50(2):132-135

7 Haider G, Kumar S, Salam B, Masood N, Jamal A, Rasheed YA. Determination of complication rate of PICC lines in oncological patients. J Pak Med Assoc 2009;59(10):663-667

\section{Certificate Course in Telemedicine Practice Guidelines}

If you want to get certified in doing Telemedicine Consultation (as per requirements of Govt of India notification), please enrol for the six module online course at:

https://www.onference.in/c/certificate-course-in-telemedicine-by-acdh/1610

for futher details please email mediclawgic@gmail.com 